\title{
Approach of Cavitary Effusion Classification and Comparison between Manual and Automatic Methods for Total Nucleated Cell Count
}

\author{
Nilson Júnior da Silva Nunes, Naila Cristina Blatt Duda, Juliana Pereira Matheus, \\ Ana Paula Soares Borenstein, Bruno Albuquerque de Almeida, Angelica Menin \& Stella de Faria Valle
}

\begin{abstract}
Background: Two classifications are used to categorize cavitary effusions using total nucleated cell count (TNCC): protein concentration and pathophysiology of its formation. The aims of the present study were to evaluate the correlation between the TNCC values of cavitary effusions obtained in the automatic and the manual method, and also evaluating the classification methodology.

Materials, Methods \& Results: Cavitary effusions were analyzed for physical, chemical and cytological aspects, as well as manual and automatic cell counts for the correlation between the traditional methods and those suggested by Stockham \& Scott. Bland-Altman regression and Spearman correlation analysis were performed. Of the total, 44 were abdominal effusions (73.3\%), 15 thoracic (25\%) and 1 pericardial (1.7\%). According to the traditional classification, most of the effusions were classified as modified transudates $(40 \%)$ and according to the classification of Stockham and Scott, as transudates poor in protein $(31.7 \%)$. The correlation between cell counting techniques between pure, modified and exudate transudates was $0.94,0.97$ and 0.94 , respectively, indicating an excellent correlation between the parameters $(P=0.95 \%)$. Discussion: Considering the concentration of proteins and CCNT, the effusions classified as modified transudate were mainly caused by neoplastic processes (carcinomas/adenocarcinomas), since there are several mechanisms of their formation, such as large variation of protein concentration. According to the Stockham \& Scott classification a unique classification is considered for exfoliative neoplastic effusions, the variation of the protein concentration of the effusion does not alter its classification. In neoplastic effusions, classified as exudates, lymphomas were the most prevalent, and hypercellularity (approximately 150,000 cells / $\mu \mathrm{L}$ ) allowed this classification. When considering low-protein transudates, the findings related to low concentrations did not differ much from the traditional classification. In the ruptures of viscera and vessels, the hemorrhagic ones were the most frequent, thus, the cytological diagnosis is essential, since it can give information about the contamination with blood during the collection. Most of these were due to neoplasia as the underlying cause. A case of chylotorax was diagnosed by comparing cholesterol and triglyceride values of effusion and serum. In cases of uroperitoneum, the presence of urine in the abdominal cavity promotes the dilution of the fluid from the cavity, being initially classified as pure transudate and, with its permanence in the cavity, increasing the CCNT, becomes an exudate. As in cases of exfoliative neoplastic effusions, the classification of the uroperitoneum, according to Stockham \& Scott, is classified directly into effusion due to rupture of the viscera, giving a quick and clear diagnosis. According to Stockham $\&$ Scott, cases classified as nonseptic exudates $(n=3)$, two of which resulted from feline infectious peritonitis (PIF). The effusive form of PIF presents with accumulations of fluid in the abdomen, having an inflammatory character, but according to the traditional classification, they enter the category of modified transudates, because, despite containing protein concentrations close to or above the serum level, they present a CCNT lower than an exudate. Cavitary effusions were classified as septic exudates when intracellular bacteria were present and in the present study, two effusions were classified as such in two patients, one with septic peritonitis and in the other the final diagnosis was not found. The high values of Spearman correlation coefficients found when comparing the automatic counts with the manual demonstrate that there is an excellent correlation between the methods and, the Bland-Altman test showed significant agreement between them.
\end{abstract}

Keywords: cell counting, cytological evaluation, cavitary fluids, flow cytometry. 


\section{INTRODUCTION}

The abnormal accumulation of fluid in the body cavities is called cavitary effusion [5] and the laboratory evaluation of this formation can help in the clinical diagnosis and it is possible to determine the etiology of its physiopathological process [5,11]. Among the causes of fluid formation in the cavities are the elevation of the hydrostatic pressure, the decrease of the oncotic pressure and the increase of vascular permeability [15]. In addition, it is known that other processes may be associated with the formation of effusions, such as extravasation of blood, lymph, urine and bile [3].

Currently in veterinary medicine, two classifications are considered in the interpretation of cavitary effusions. The traditional classification, widely used, divides fluids into pure transudates, modified transudates and exudates, not taking into consideration the mechanism of liquid formation. The classification suggested by Stockham and Scott [12], divides effusions according to the pathophysiological mechanism: in protein-poor $(\leq 2.5 \mathrm{~g} / \mathrm{dL})$ and protein-rich transudates $(>2.5 \mathrm{~g} / \mathrm{dL})$, septic and nonseptic exudates, effusions due to ruptures of vessels and / or viscera, neoplastic effusions and effusions caused by reactive mesothelial proliferation $[12,15]$.

In the laboratory, effusions are analyzed for their physical, chemical and cellular characteristics, and the total nucleated cell count (TNCC) plays an important role in classification and interpretation [3]. For this analysis, most veterinary laboratories adopt manual counting in the hemocytometer (Neubauer chamber) in the laboratory routine, and some laboratories do not perform this count. Currently, some hematology counters are programmed to perform this analysis. Supposedly, this electronic analysis allows a greater accuracy in the determination of the TNCC, but there are no studies that correlate both techniques.

The objectives of the present study were to evaluate two methodologies for classification of cavitary effusions in veterinary medicine and to correlate the TNCC obtained by manual methodology in Neubauer chamber and the automatic methodology.

\section{MATERIALS AND METHODS}

\section{Samples}

Samples of pericardial, thoracic and abdominal effusions from 60 animals (35 dogs, 21 cats and
4 horses) from the routine of the Hospital de Clínicas Veterinárias of the Federal University of Rio Grande do Sul (HCV-UFRGS) were analyzed and sent to Laboratory of Clinical Veterinary Analyzes (LACVet) for diagnostic purposes. All samples were received in tubes with or without anticoagulant EDTA- $\mathrm{K}_{2}{ }^{1}$ for evaluation of TNCC, $\mathrm{pH}$, density and cytological evaluation and, in tubes without anticoagulant, for biochemical analyzes that were performed as needed. The criteria for inclusion of the samples in the study were sufficient volume for all tests, absence of grumus and / or clots, and sample volume with right proportion of blood and anticoagulant. In addition, samples were included in which there were in parallel blood samples for blood counts and biochemical tests.

\section{Processing of effusion samples}

All samples were processed immediately upon receipt in the laboratory, regardless of species and site of fluid formation. At first, the samples in anticoagulant tubes were analyzed in the IDEXX ProCyte $\mathrm{Dx}^{2}$ automatic hematology analyzer for TNCC. With the same sample, the manual counting was performed in the Neubauer chamber in duplicate, and the final result was obtained through the mean of the two counts.

For the cytological evaluation of the effusions, 4 smears (squash type) were performed, two with the pure sample and two after centrifugation for $10 \mathrm{~min}$ at $1,200 \mathrm{~g}$. At least one of the slides were stained with Diff-quick staining ${ }^{3}$ and one with Wright dye ${ }^{4}$. Some slides were left unstained if additional stains were needed. All stained slides were evaluated under an optical microscope, by the same observer, in 10x, 40x and 100x magnification lenses (with immersion).

In those effusions that had a hemorrhagic appearance, the hematocrit was determined by the microhematocrit technique (5 min at 8,200 $\mathrm{g}$ ) and compared to the hematocrit value of the peripheral blood. For the determination of the protein values, the colorimetric method (biuret) was used and in the cases of effusions suggestive of FIP (feline infectious peritonitis), the Rivalta test was performed.

For the biochemical analysis, the samples without anticoagulant were centrifuged ( $5 \mathrm{~min}$ at 2,240 $\mathrm{g}$ ), the supernatants were separated and used for albumin, creatinine, triglycerides, total protein, cholesterol and glucose evaluation by kinetic assay in a CM 200 automatic biochemical analyzer according to the manu- 
facturer's recommendations. The same determinations were performed on the peripheral blood samples.

\section{Effusions classification}

After the physical, chemical and cytological analyzes, the samples were first distributed according to the traditional classification in pure transudates (TNCC $\leq 1,000$ cells / $\mu \mathrm{L}$ and proteins $<2.5 \mathrm{~g} / \mathrm{dL}$ ), modified transudates (TNCC of 1,000 to 7,000 cells / $\mu \mathrm{L}$ and proteins $\geq 2.5$ and $<3.0 \mathrm{~g} / \mathrm{dL}$ ) and exudates (TNCC $\geq 7000$ cells $/ \mu \mathrm{L}$ and proteins $\geq 3.0 \mathrm{~g} / \mathrm{dL}$ ) [3]. Then, the samples were reclassified according to the classification suggested by Stockham \& Scott [12], in protein-poor (protein $<2.5 \mathrm{~g} / \mathrm{dL}$ ) and protein-rich transudates (proteins $\geq 2.5 \mathrm{~g} / \mathrm{dL}$ ), septic exudates or nonseptic, considering the presence or absence of bacteria in cytological evaluation, neoplastic exfoliative effusions, effusions due to rupture of vessels and / or rupture of viscera.

\section{Statistical Analysis}

Before performing simple linear regression, the TNCC measurement data by manual and automatic methodology were analyzed to verify if they met the assumptions of the model. The data were submitted to the Shapiro-Wilk, Levene, Durbin Watson and Pearson correlation tests for normality, homogeneity of variance, residue independence and correlation, respectively. Furthermore, the data were analyzed using the Bland-Altman test, followed by a simple linear regression of the difference between the methods and the average between them. Concordance limits were calculated using the $95 \%$ range (mean \pm 1.96 standard deviation). The level of significance used for rejection of the null hypothesis was $5 \%(P \leq 0.05)$. Statistical analyzes were conducted using IBM SPSS Statistics for Windows software, version 22.0 (IBM Corp. Armonk, 2013).

\section{RESULTS}

A total of 93 samples of cavitary effusions were analyzed, and 33 (35.4\%) were excluded because they did not meet the inclusion criteria. Out of the analyzed samples, 44 were abdominal effusions $(73.3 \%), 15$ thoracic $(25 \%)$ and 1 pericardial $(1.7 \%)$ which were classified, according to the traditional classification in modified transudates $(24 / 60,40 \%)$, exudates $(23 / 60$, $38.33 \%$ ) and pure transudates $(13 / 60,21.67 \%)$. In the classification suggested by Stockham and Scott [12], effusions were categorized as protein-poor transudates $(19 / 60,31.7 \%)$, protein-rich transudates $(13 / 60$, $21.6 \%)$, neoplastic exfoliative effusions $(11 / 60,18.3 \%$ ), effusions due to vessel rupture $(7 / 60,11.7 \%)$, effusions due to rupture of viscera $(5 / 60,8.3 \%)$, nonseptic exudates $(3 / 60,5 \%)$, and septic exudates $(2 / 60 ; 3.3 \%)$. Biochemical analyzes of both peripheral blood and effusions were performed according to need and classifications (Tables 1 and 2). The etiologies were diverse and were directed to both classifications (Table 3).

Table 1. Results of concentrations of albumin, globulin, creatinine, triglycerides, total protein, cholesterol and glucose determined from effusion determined by kinetic assay.

\begin{tabular}{cccc}
\hline Parameter & $\mathrm{n}$ & Reference value & Median, min-max and average \\
\hline Albumin $(\mathrm{g} / \mathrm{dL})$ & 53 & $2.6-3.3$ & $1.4(0-5) 1.68$ \\
Cholesterol $(\mathrm{mg} / \mathrm{dL})$ & 54 & $135-270$ & $54(0-192) 55.53$ \\
Creatinine $(\mathrm{mg} / \mathrm{dL})$ & 54 & $0.5-1.5$ & $0.73(0.23-116.2) 4.39$ \\
Glucose $(\mathrm{mg} / \mathrm{dL})$ & 57 & $65-118$ & $105(10-207) 100.5$ \\
Globulin $(\mathrm{g} / \mathrm{dL})$ & 57 & $2.7-4.4$ & $1.9(0.2-11) 2.25$ \\
Total Protein $(\mathrm{g} / \mathrm{dL})$ & 56 & $5.4-7.1$ & $3.6(0.3-15) 3.84$ \\
Triglycerides $(\mathrm{mg} / \mathrm{dL})$ & 55 & $32-138$ & $18(0-620) 43.83$ \\
\hline
\end{tabular}

Table 2. Results of albumin, globulin, creatinine, triglycerides, total protein, cholesterol and glucose serum concentrations determined by kinetic assay.

\begin{tabular}{cccc}
\hline Parameter & $\mathrm{n}$ & Reference values & Median, min-max and average \\
\hline Albumin $(\mathrm{g} / \mathrm{dL})$ & 53 & $2.6-3.3$ & $2.6(2-5.2) 2.65$ \\
Cholesterol $(\mathrm{mg} / \mathrm{dL})$ & 54 & $135-270$ & $157.5(28-298) 163$ \\
Creatinine $(\mathrm{mg} / \mathrm{dL})$ & 54 & $0.5-1.5$ & $0.72(0.21-28.0) 2.03$ \\
Glucose $(\mathrm{mg} / \mathrm{dL})$ & 52 & $65-118$ & $98(44-205) 103.2$ \\
Globulin $(\mathrm{g} / \mathrm{dL})$ & 57 & $2.7-4.4$ & $3.65(1.2-9.5) 3.71$ \\
Total Protein $(\mathrm{g} / \mathrm{dL})$ & 56 & $5.4-7.1$ & $6.7(2.4-12.3) 6.37$ \\
Triglycerides $(\mathrm{mg} / \mathrm{dL})$ & 55 & $32-138$ & $63.5(3-2184) 143$ \\
\hline
\end{tabular}


N.J.S. Nunes, N.C.B. Duda, J.P. Matheus, et al. 2018. Approach of Cavitary Effusion Classification and Comparison between Manual and Automatic Methods for Total Nucleated Cell Count.

Acta Scientiae Veterinariae. 46: 1582.

Tabela 3. Classification of effusion according to their etiology based on the traditional classification and the classification suggested by Stockham and Scott*.

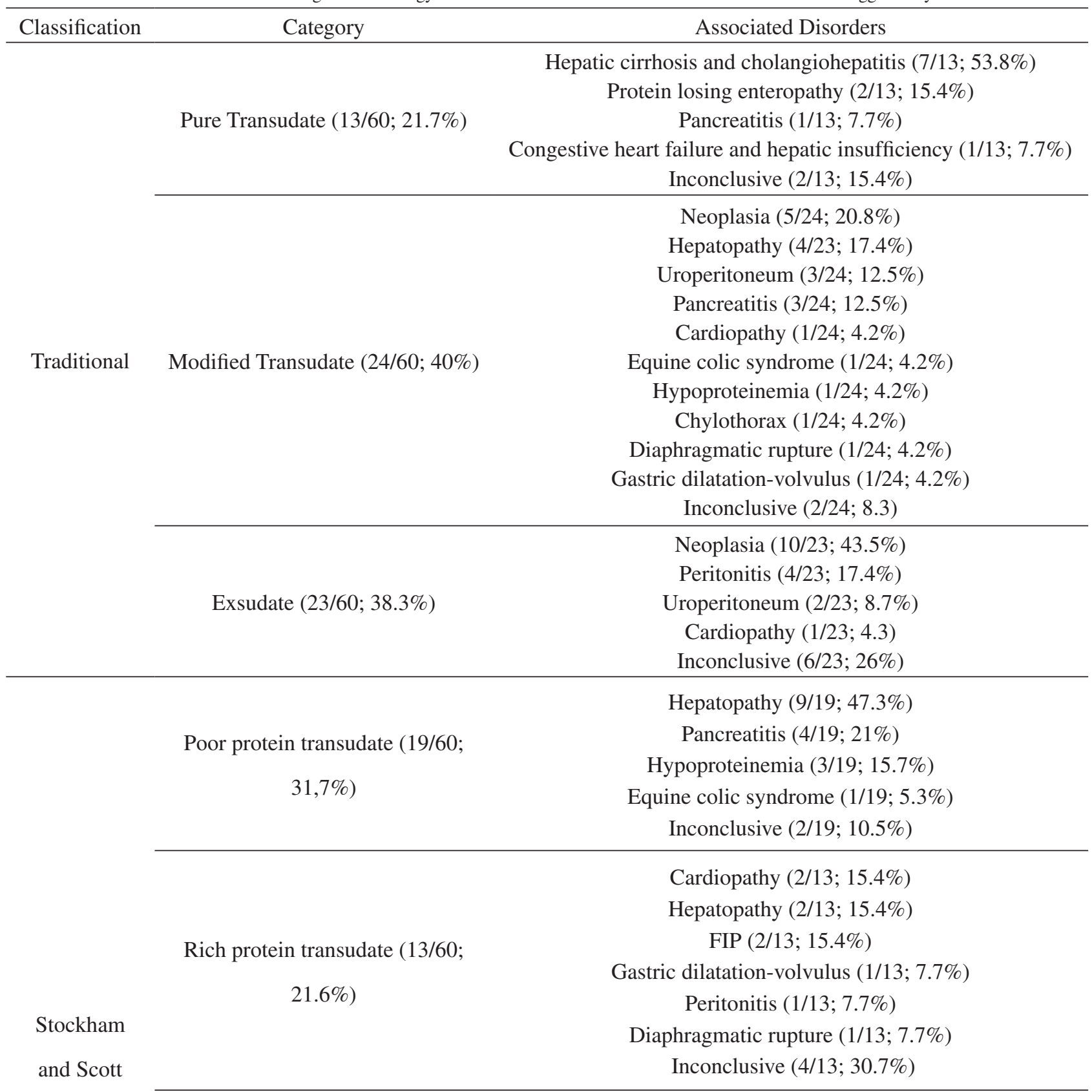

\begin{tabular}{cc}
\hline Septic exsudate $(2 / 60 ; 3.3 \%)$ & Peritonitis $(1 / 2 ; 50 \%)$ \\
Inconclusive $(1 / 2 ; 50 \%)$ \\
\hline Non-septic exsudate $(3 / 60 ; 5 \%)$ & FIP $(2 / 3 ; 66.6 \%)$ \\
Peritonitis $(1 / 3 ; 33.3 \%)$
\end{tabular}

Neoplasia $(11 / 60 ; 18.3 \%)$

Linfoproliferative $(6 / 11 ; 54.5 \%)$

Carcinoma or adenoma $(5 / 11 ; 45.4 \%)$

\begin{tabular}{cc}
\hline Vessels rupture $(7 / 60 ; 11.7 \%)$ & $\begin{array}{c}\text { Hemorrhagic effusion }(6 / 7 ; 85.7 \%) \\
\text { Chylous effusion }(1 / 7 ; 14.3 \%)\end{array}$ \\
\hline Viscera rupture $(5 / 60 ; 8.3 \%)$ & Uroperitoneum $(5 / 5 ; 100 \%)$ \\
\hline
\end{tabular}

*Stockham S.L. \& Scott M.A. 2008. Fundamentals of Veterinary Clinical Pathology. [12]. 
The results of TNCC obtained using a manual and automatic method, used in the linear regression analysis, had a normal distribution $(P=0.054)$, homoscedasticity $(P=0.996)$, independence $(\mathrm{DW}=$ $2.144)$ and presented a positive correlation $(P=0.000)$. According to the simple linear regression analysis, it is possible to observe that the proposed mathematical model explains $98 \%\left(\mathrm{R}^{2}=0.989\right)$ of the variation found in the TNCC evaluated by the automatic method. The equation of the mathematical model, $\mathrm{y}=2.49 * 102$ $+1.01 * x$, "y" equal to the TNCC evaluated by the automatic method and the " $\mathrm{x}$ " corresponding to the TNCC evaluated by the manual method.

Through the Bland-Altman analysis, it was possible to observe that the boundaries of difference appear broad for counts below 15,000 nucleated cells, and small for counts above this value, demonstrating a clear relation between the difference between the two methods and their mean, with the difference between methods increasing according to the TNCC increase. Although the average demonstrates that the automatic method measured around 375 cells more than the manual method, a linear regression of the difference between methods with the mean shows that the mean values are not different from zero $(P=0.306)$, there being no bias. The $\mathrm{R} 2$ equals 0.001 shows that the mean values are not related, and only explain $0.1 \%$ of the mean variance of the difference between measurements by the automatic and manual method.

\section{DISCUSSION}

Through the effusion classification, which must be described in laboratory reports, the clinician may request additional tests that are effective in determining the cause of the formation. Considering the physical, chemical, TNCC and cellular composition, the interpretations of the types of effusions analyzed in the present study varied between the two classifications studied.

Based in the criteria for classification which considers the proteins values and TNCC, effusions classified as modified transudates were mostly caused by neoplastic processes and liver diseases. Among the neoplasia observed in the analyzed effusions, epithelial neoplasia (carcinomas / adenocarcinomas) presented a higher occurrence. The classification of those as modified transudates in the traditional classification is pertinent because the mechanism of effusion formation can be varied, occurring by hemorrhage, compression of lymphatic vessels, inflammation and necrosis. In clinical practice, this type of classification may suggest a less serious etiology and cause doubts as to the interpretation and direction of the complementary exams. In the classification suggested by Stockham and Scott [12], these effusions are classified as neoplastic effusions, directing the clinical management for additional complementary tests that confirm the etiology. In the present study, carcinomas and adenocarcinomas were the most frequent neoplasias in liquids classified as modified transudates, as previously described in the literature [3].

Another type of neoplastic effusion, those caused by round cells, may have different classifications between the traditional and the suggested by Stockham and Scott [12]. In traditional, those effusions were classified as exudates, due to hypercellularity (TNCC mean of 150,000 cells / $\mu \mathrm{L}$ ). In general, these neoplasias are caused by lymphomas, which due to the origin of round cells, exfoliate a large number of cells [8]. Exudates, thus classified by high TNCC, can also be caused by processes that cause direct irritation to the abdominal cavity, leading to the migration of inflammatory cells and to the accumulation of a secondary non-septic effusion or to become septic over time [1]. In both classifications, due to intense cellularity, the evaluation should include the predominant cell type as well as the verification of the cellular morphological alterations, to help the interpretation of the exam.

In the protein-poor transudates, the findings related to low protein concentration and poor cellularity did not differ much from the traditional classification of pure transudate. This result suggests that, regardless of the classification of this type of effusion, it will not cause a diagnosis change. In any case, the classification suggested by Stockham and Scott [12] is still more advantageous because it reports a more probable etiology. The effusions of these classifications, observed in the present study, were liver diseases that result in low oncotic pressure due to the reduction of albumin synthesis by the liver. However, concomitantly, it is necessary to evaluate the presence of neoplasias underlying liver disease and the presence of metastasis in the patients [14].

In the effusions classified as neoplastic exfoliative, in the traditional classification, some of them were classified as modified transudate due to the great 
variability of the protein values since the mechanisms of formation are multifactorial and the cellularity depends on the ability of the exfoliative neoplasia cells in the effusion. Due to the fact that the diagnostic and mainly therapeutic approach to neoplastic effusions requires careful management, TNCC and additional tests [16] (such as special stains) should be considered in the classification and interpretation of classified liquids. As the classification of Stockham and Scott [12] considers a unique classification for exfoliative neoplastic effusions, and the variation of the protein concentration of the effusion does not alter its classification, the information passed to the veterinarian becomes more effective.

In cases of effusions caused by rupture of viscera and vessels, hemorrhagic effusions were the most frequent, and the mean hematocrit was $\geq 10 \%$. In these cases, the cytological diagnosis is essential, because from this analysis, it is possible to differentiate from iatrogenic contamination of the collection. Out of the 6 hemorrhagic effusions evaluated, $66.7 \%$ were due to neoplasias as the underlying cause, and $33.3 \%$ did not have their etiology confirmed. The presence of hemothorax and hemoabdomen is common as a consequence of some neoplasias, as in cases of hemangiosarcoma, mesothelioma and metastatic carcinoma [9].

One of the effusions evaluated was due to the rupture of the thoracic duct (chylotorax), identified by the comparison of cholesterol and triglyceride values of effusion and serum. In this case, the values of cholesterol in the liquid were lower than in the serum, unlike the triglycerides that were higher than the serum concentrations [8]. Additional biochemical tests are critical for this type of effusion since chylous effusion can be easily mistaken for pseudochylous and lymphomatous effusions as a result of thoracic lymphoma.

In 5 analyzed samples, it was possible to detect the presence of uroperitoneum (8.3\%), caused by ruptures in the urinary tract due to traumas or urinary obstructions [2]. The presence of urine in the abdominal cavity promotes the dilution of the liquid in the cavity, which is classified as pure transudate immediately after the rupture, due to the decrease in protein concentration and cellularity. Over time, a secondary inflammatory process occurs, elevating the TNCC and transforming the effusion into an exudate. In the analyzed samples, serum creatinine and effusion were measured to confirm the diagnosis of uroperitoneum, which should be greater than 2 times the serum creatinine concentration to confirm [8]. As in cases of exfoliative neoplastic effusions, the classification of the uroperitoneum, according to Stockham and Scott [12], is classified as effusion due to rupture of the viscus, giving a clear diagnosis for the beginning of the surgical treatment.

According to the classification suggested by Stockham and Scott [12], 3 cases were classified as nonseptic exudates, 2 cases resulting from feline infectious peritonitis (FIP) and 1 case of pancreatitis. The effusive form of FIP presents itself with accumulation of fluid in the thoracic regions and, more frequently, in the abdominal region [10]. Cavitary effusions due to PIF have an inflammatory character, but, according to the traditional classification, often fall into the category of modified transudates, due to the fact that, although it contains protein concentrations close to or above the serum level, they present a TNCC lower than an exudate [17]. The Rivalta test, which was positive for the analyzed effusions, is a subjective method for detecting FIP and is not sensitive and specific for diagnosis $[6,13]$. However, effusions caused by other inflammatory causes, which are not due to FIP, may be positive in the Rivalta test. Therefore, a careful evaluation of the interpretation of the result of this test must be performed.

Often, septic effusions are caused by bacterial infections related to secondary infections such as penetrating wounds, foreign bodies, translocations of microorganisms via lung, gastrointestinal and hematogenous tissue [5]. Septic exudates were verified in two samples and in cytology, intracellular bacteria were present. the combination of cytological examination of effusion and aerobic and anaerobic bacterial cultures are important for the identification of microorganisms [4] since if the patient is receiving antimicrobials, the evidence of the bacteria cannot be visualized in cytology.

The TNCC plays an important role in the classification and interpretation of cavity liquids, and although manual counting in the Neubauer chamber is the gold standard, automatic counting can be performed. Automatic hematology counters that have optical fluorescence technology are able to differentiate nucleated cells based on the complexity and amount of RNA [7]. The high values of Spearman's correlation coefficients found, when comparing automatic counts with the manual method, show that there is an excel- 
lent correlation between the methods and that the small difference between the counts will not interfere in the classification and diagnosis of the effusion. The BlandAltman test also demonstrated significant agreement between manual and automatic methods, suggesting that the hematology counter with single flow cytometry can be safely used for TNCC.

\section{CONCLUSION}

Considering the present study, it can be affirmed that there is a correlation between the techniques of evaluation of the cellular counts and the automatic counts, being able to be used safely, due to their excellent degree of correlation. It is also concluded that the quantitative, qualitative and interpretative analyzes of the effusions help in the diagnosis of specific diseases, making it a routine evaluation. However, they should not be classified using only the evaluation of an isolated parameter, such as only protein, TNCC or biochemical analyzes. In addition, there is some variation between the classifications of effusions, and the one that provides a more accurate diagnosis of the formation etiology, which is the classification suggested by Stockham and Scott, should be used.

\section{MANUFACTURERS}

${ }^{1}$ BD Vacutainer. Franklin Lakes, NJ, USA.

${ }^{2}$ Idexx Laboratories. Westbrook, ME, USA.

${ }^{3}$ Newprov Produtos para Laboratório. Pinhais, PR, Brazil.

${ }^{4}$ Laborclin Produtos para Laboratórios Ltda. Pinhais, PR, Brazil.

${ }^{5}$ Wiener Lab Group. Rosário, Santa Fé, Argentina.

Declaration of interest. The authors report no conflicts of interest. The authors alone are responsible for the content and writing of the paper.

\section{REFERENCES}

1 Alleman A.R. 2003. Abdominal, thoracic, and pericardial effusions. Veterinary Clinics of North American: Small Animal Practice. 33: 89-118.

2 Balakrishnan A. \& Drobatz K.J. 2013. Management of urinary tract emergencies in small animals. Veterinary Clinics of North American: Small Animal Practice. 43: 843-867.

3 Bohn A.A. 2017. Analysis of canine peritoneal fluid analysis. Veterinary Clinics of North American: Small Animal Practice. 47: 123-133.

4 Bonczynski J., Ludwig L.L., Barton L.J., Loar A. \& Peterson M.E. 2003. Comparison of peritoneal fluid and peripheral blood $\mathrm{pH}$, bicarbonate, glucose, and lactate concentration as a diagnostic tool for septic peritonitis in dogs and cats. Veterinary Surgery. 32: 161-166.

5 Dempsey S.M. \& Ewing P.J. 2011. A review of the pathophysiology, classification, and analysis of canine and feline cavitary effusions. Journal of the American Animal Hospital Association. 47: 1-11.

6 Fischer Y., Sauter-Louis C. \& Hartmann K. 2012. Diagnostic accuracy of the Rivalta test for 793 feline infectious peritonitis. Veterinary Clinical Pathology. 41: 558-567.

7 Goldmann F., Bauer N. \& Moritz A. 2014. Evaluation of the IDEXX ProCyte Dx analyzer for dogs and cats compared to the Siemens ADVIA 2120 and manual differential. Comparative Clinical Pathology. 23: 283-296.

8 Hetzel N., Papasouliotis K., Dodkin S. \& Murphy K. 2012. Biochemical assessment of canine body cavity effusions using three bench-top analyzers. Journal of the Small Animal Practice. 53: 459-64.

9 Nakamura R.K., Rozanski E.A. \& Rush J.E. 2008. Non-coagulopathic spontaneous hemothorax in dogs. Journal of Veterinary Emergency and Critical Care. 18: 292-297.

10 Ritz S., Egberink H. \& Hartmann K. 2007. Effect of feline interferon-omega on the survival time and quality of life of cats with feline infectious peritonitis. Journal of Veterinary Internal Medicine. 21: 1193-1197.

11 Rose A., Funk D. \& Neiger R. 2016. Comparison of refractometry and biuret assay for measurement of total protein concentration in canine abdominal and pleural fluid specimens. Journal of the American Veterinary Medical Association. 248: 789-794.

12 Stockham S.L. \& Scott M.A. 2008. Cavitary effusions. In: Fundamentals of Veterinary Clinical Pathology. 2nd edn. Ames: Blackwell Publishing, pp.831-868.

13 Tasker S. 2018. Diagnosis of feline infectious peritonitis: Update on evidence supporting available tests. Journal of Feline Medicine and Surgery. 20: 228-243. 
14 Thawley V. 2017. Acute liver injury and failure. Veterinary Clinics of North American: Small Animal Practice. 47 : 617-630.

15 Thompson C.A. \& Rebar A.H. 2016. Body cavity fluids. In: Raskin R.E. \& Meyer D.J. (Eds). Canine and feline cytology: a color atlas and interpretation guide. 3rd edn. St. Louis: Elsevier Saunders, pp.191-294.

16 Wallace K.A., Goldschmidt M.H. \& Patel R.T. 2015. Converting fluid-based cytologic specimens to histologic specimens for immunohistochemistry. Veterinary Clinical Pathology. 44: 303-309.

17 Zoia A., Slater L.A., Heller J., Connolly D.J. \& Church D.B. 2009. A new approach to pleural effusion in cats: markers for distinguishing transudates from exudates. Journal of Feline Medicine and Surgery. 11: 847-855. 